\title{
Design of Lane Departure Warning System Based on ADSP-BF561 Yongtao Liu $^{\mathrm{a}}$, Lang Wei ${ }^{\mathrm{b}}$ and Jie Qiao ${ }^{\mathrm{C}}$ Department of Vehicle Engineering, Chang'an University, Xi'an, China aliuyongtao86@163.com, bqc_1@chd.edu.cn, cqiaojie88@163.com
}

Keywords: lane departure warning system, ADSP-BF561 processor, vision sensor, image processing

\begin{abstract}
Aiming at the problem of more and more traffic accidents which are caused by the driver behaviors, this paper designs a lane departure warning system based on the ADSP-BF561 processor. Vision sensor is used to capture road images, while the driving parameters are collected by the microprocessor. The lane marking detection speed can be more than 25 frames per second. The system is equipped with the test vehicle for hundreds of kilometers on highway, and the results show that this system has a better efficiency and feasibility.
\end{abstract}

\section{Introduction}

With a large increase of the vehicles all over the world, the traffic transportation safety problems have attracted more and more people's extensive attention. According to statistics, $80 \%$ of the traffic accidents were caused by the driver's subjective factors, such as driver distraction, negligence, and fatigue [1]. If the warning is given one second ahead of danger, $90 \%$ of traffic accidents can be avoided [2]. Thus, reducing traffic accidents caused by the driver factors is the focus of the transportation security. On one hand, drivers need to strengthen their literacy of traffic rules; on the other hand, technical measures should be taken on the driver's dangerous behavior for early warning. The lane departure warning system installed on the vehicle is a very effective way [3].

The traditional lane departure warning systems are based on IPC platforms, which are not only bulky and costly, but also not suitable for marking. In this paper, we design a lane departure warning system based on the vision sensor and ADSP-BF561 processor, which has a small, high processing speed. The system will not only be able to solve some illegal behaviors, such as driving across the lane, but also can provide detailed information for the traffic management department to restore the scene of the accident [4].

\section{System Overview}

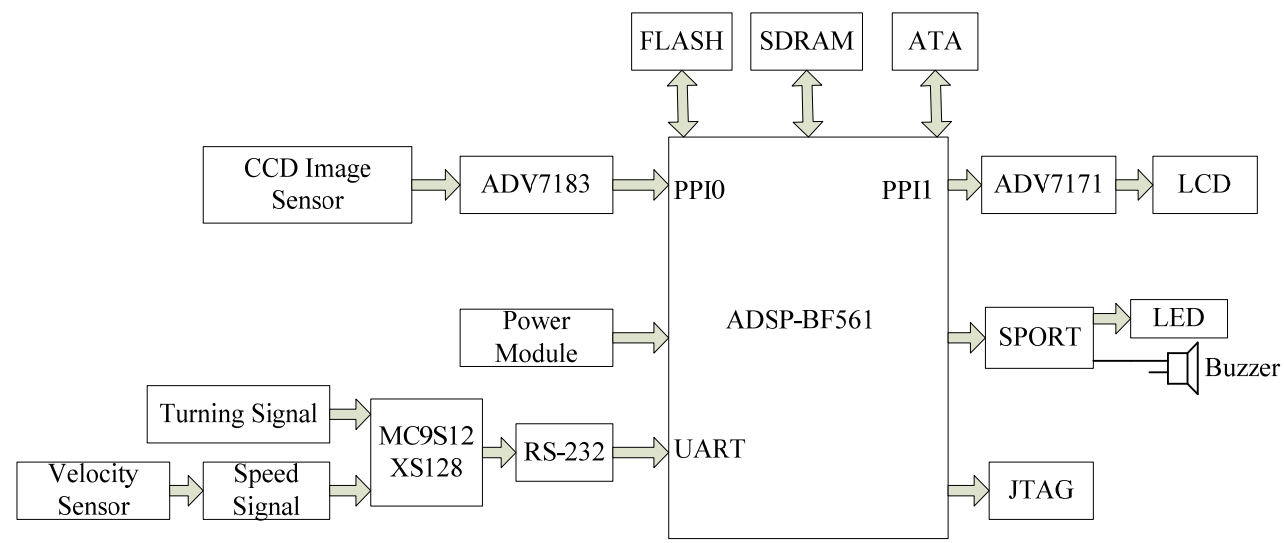

Fig. 1 Hardware design of the system

Hardware Architecture. As shown in Fig. 1, this system is mainly achieved through the ADSP-BF561, including image information acquisition and image processing. Meanwhile, in order to improve the efficiency of the system, MC9S12XS128 SCM is used as the turn signal and speed signal processing unit. The CCD image sensor is used as the vision sensor to obtain the lane image, 
while the video image can be shown in the LCD display. When the lane departure occurs, the system can give a sound and light warning, and store the illegal image into the hard disk.

Software Workflow. The software mainly consists of three parts, such as graying module, median filter module and binarization module $[5,6]$. Then the processor will detect lanes and the position of the vehicle in the image is calculated. According to the lane departure warning model combined with the turn signal, the system will determine whether the warning should be happened or not.

\section{Hardware Design}

ADSP-BF561 Processor. ADSP-BF561 processor has a better performance in the Blackfin family [7]. Features are as follows:

- Dual symmetric $600 \mathrm{MHz}$ high performance cores, each core includes two 16-bit MACs.

- $328 \mathrm{~K}$ bytes of on-chip memory, 2 parallel input/output peripheral interface units, 12 general-purpose 32-bit times/counters with PWM.

Vision sensor. Vision sensor adopts the color camera WAT-231S2 with its dimensions of 1/3 inch. Due to its PAL scanning type, the sensor is not sensitive to the phase distortion. Some features of the vision sensor are as follows: 400,000 pixels, CS interface, and the SNR is 50db, the camera is initialized by the VDSP++ software development environment. As a result, a clear image can be obtained through this kind of vision sensor even in a high-speed vehicle.

The capture and output system of video image.

Image capture. In this system, the CCD camera is used as an image acquisition device, but it outputs analog signals, which cannot be directly processed by the DSP. Thus, the image decoder chip ADV7183 can be used to convert the analog signal to the digital signal. Fig. 2 shows the general connections from the ADV7183 to the ADSP-BF561.

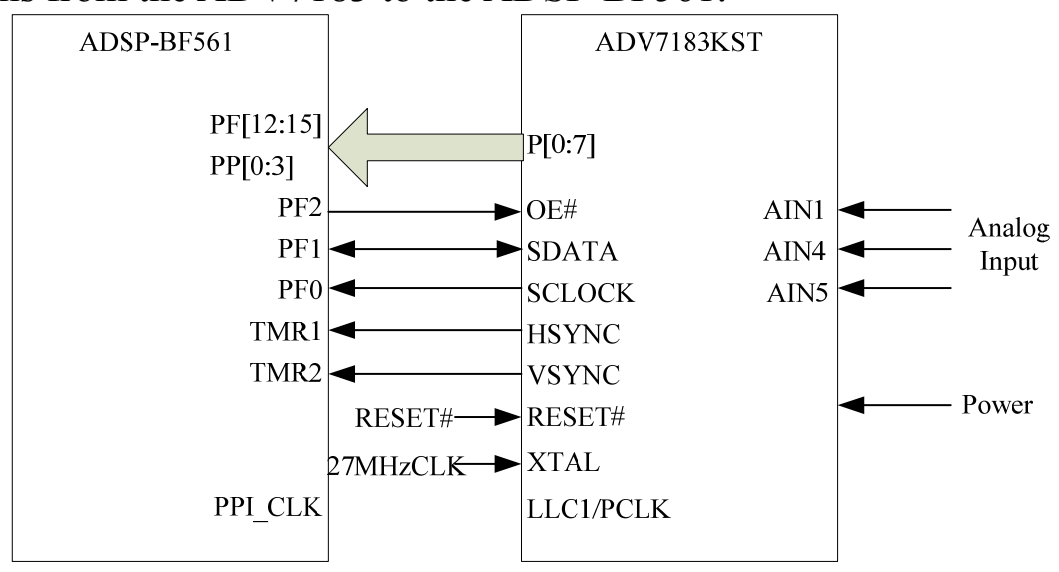

Fig. 2 Connections from ADV7183 to ADSP-BF561

Image output. In order to display the result of the lane detection, we add a TVG-800 LCD to the system. The system adopts the ADV7171 video encoder chip to acquire an analog signal output to the LCD display. The ADV7171 provides a three-way video analog signal output channels, and it can directly connect to the ADSP-BF561 through the PPI. Fig. 3 shows a block diagram of the basic connection between the BF561 and an ADV7171.

Vehicle parameters acquisition system. The system uses a voltage comparator LM311 and its peripheral circuits to conduct opt-coupler isolation. The processed signal can be transmitted to the PAD0 pin of the MC9S12XS128 high performance microcontroller.

Turn light line under the dashboard can provide turn signal, which will be transferred to the PAD2 of the microcontroller after opt- coupler isolation.

Image storage. This part connects with the 160G ATA hard disk through a standard IDE interface, in order to store a number of the violation images.

Sound and light warning. This part mainly includes electronic components such as some light-emitting diodes and buzzers. 


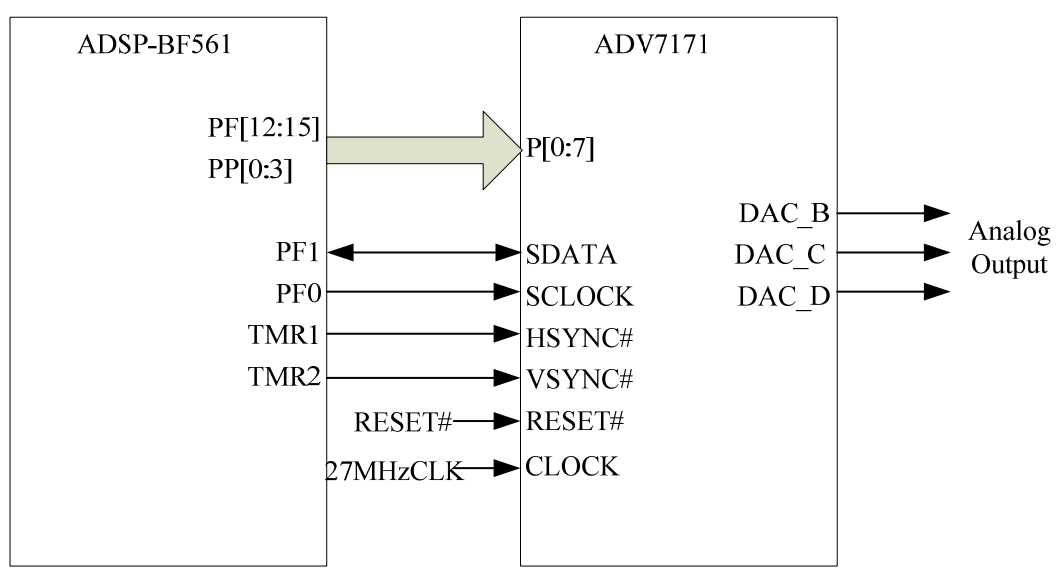

Fig. 3 Connections between ADSP-BF561 and ADV7171

\section{Analysis of Experimental Results}

System structures. The vision sensor is installed on the central location inside the front windshield of an IVECO automobile NJ6400GHA3, with $1.7 \mathrm{~m}$ height and pitch angle of $14.5^{\circ}$.

Inter-calibration parameters. Through the Mat-lab Camera Calibration Toolbox developed by the Caltech Vision Lab, the camera's internal parameters are obtained, as shown in Table 1.

Table 1 The camera's internal parameters

\begin{tabular}{cccc}
\hline Parameter Name & Horizontal Focal Length & Vertical Focal Length & Principal Point [U,V] \\
\hline Value & 719.1 & 719.3 & {$[318.1,235.2]$} \\
Error Estimate & 3.8 & 3.7 & {$[1.5,2.1]$}
\end{tabular}

Experimental results. The system is equipped on an IVECO automobile and we do some experiments on a straight road. The results show that solid and dashed marking line can be well identified. In addition, it also has good recognition results during day and night, as shown in Fig. 4. Among the hundreds of illegal images, the recognition accuracy rate reaches more than $90 \%$.

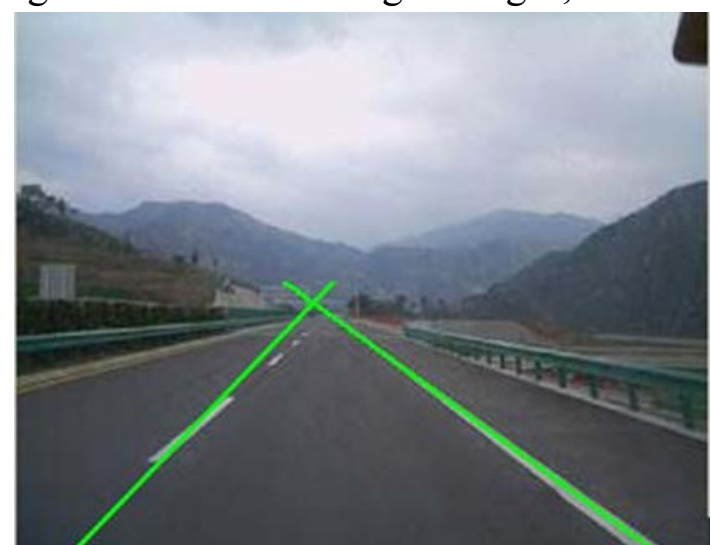

a) Day condition

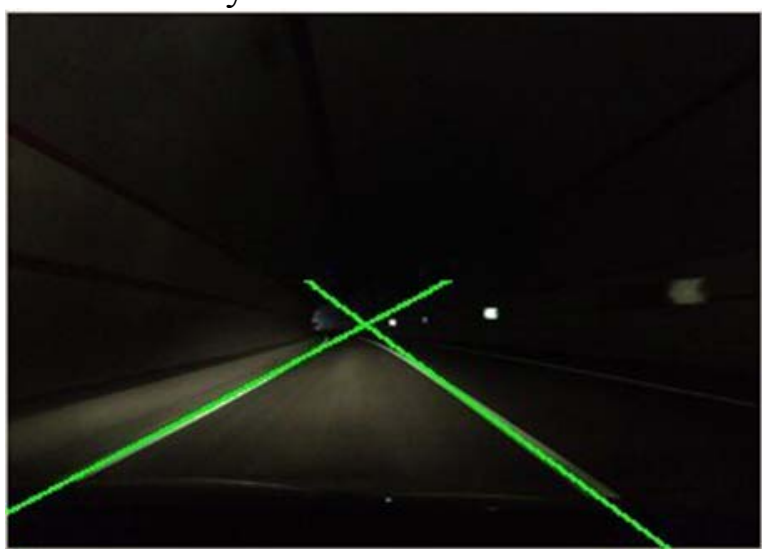

b) Night condition

Fig. 4 Test results

\section{Conclusion}

This paper analyzes the hardware structure and work process about a lane departure warning system based on the ADSP-BF561 processor. After the road test, it works reliably under different conditions. Due to its low cost and small size, it will have a broad market prospect.

\section{Acknowledgement}

This research is sponsored by National Nature Science Foundation of China, under the contract number 51278062 . 


\section{References}

[1] LIN Guang-yu, WEI Lang: Air Force Engineering University Journal(natural science). Vol. 7(5) (2006), p. 30-32

[2] Zhu Yun-jiang, Fan Gang, Wu Dong: The 2011 International Conference on Transportation, Mechanical, and Electrical Engineering. (2011), p. 476-480

[3] LIN Guang-yu, WEI Lang: The 2009 IEEE International Conference on Mechatronics and Automation. (2009), p. 4991-4996

[4] Xinguang Du, Xianlong Jin, Xiaoyun Zhang, Jie Shen, Xinyi Hou: Advances in Engineering Software. Vol. 40(7) (2009), p. 497-505

[5] Joon Woong Lee: Computer Vision and Image Understanding. Vol. 86 (2002), p. 52-78

[6] Kunsoo Huh, Jaehak Park, Junyeon Hwang, Daegun Hong: Optics and Lasers in Engineering. Vol. 46(1) (2008), p. 168-178

[7] Information on http://www.analog.com 